\title{
FATORES DE RISCO E MEDIDAS PREVENTIVAS PARA ÚLCERA DE PRESSÃO NO LESADO MEDULAR. EXPERIÊNCIA DA EQUIPE DE ENFERMAGEM DO HCFMRP-USP*
}

\author{
RISK FACTORS AND PREVENTION OF PRESSURE ULCER IN SPINE CORD INJURY PATIENTS. \\ EXPERIENCE OF MEMBERS OF NURSING TEAM AT FMRP - USP HOSPITAL
}

\author{
Paula Cristina Nogueira ${ }^{1}$; Maria Helena L. Caliri² \& Cláudia B. dos Santos²
}

\footnotetext{
${ }^{4}$ Enfermeira da Clínica Cirúrgica do Hospital das Clínicas da Faculdade de Medicina de Ribeirão Preto - USP. ${ }^{2}$ Docentes. da Escola de Enfermagem de Ribeirão Preto - USP.

CORRESPONDENACIA: Profa. Dra. Maria Helena L. Caliri- Departamento de Enfermagem Geral e Especializada da Escola de Enfermagem de Ribeirão Preto - USP - Av. Bandeirantes 3900, cep:14040-902 - Ribeirão Preto-SP. e-mail: mhcaliri@glete.eerp.usp.br
}

NOGUEIRA PC; CALIRI MHL \& SANTOS CB. Fatores de risco e medidas preventivas para ulcera de pressão no lesado medular. experiência da equipe de enfermagem do Hospital das Clínicas da FMRP-USP. Medicina, Ribeirão Preto, 35: 14-23, jan./mar. 2002.

RESUMO: As úlceras de pressão (UP) são freqüentes no lesado medular, podendo trazer sérias complicações, como osteomielite, septicemia, amputações, e mesmo levar o paciente a óbito. Interferem na qualidade de vida do paciente e família, impedindo ou dificultando o acesso a programas de reabilitação. Assim, a prevenção e o tratamento no estágio inicial devem ser a meta da assistência que vise à qualidade. Este estudo teve como objetivo identificar as causas de UP em pacientes com traumatismos da medula espinhal, atendidos em uma instituição hospitalar, e descrever as medidas identificadas como mais importantes para a prevenção do problema, segundo a opinião de enfermeiros, auxiliares e técnicos de enfermagem. Através de um inquérito, os dados foram coletados, utilizando-se um instrumento composto de 35 frases com descritores dos fatores de risco e 18 frases com descritores de medidas preventivas, construído a partir de dados da literatura e validado anteriormente. Foi solicitado aos 85 participantes que atribuíssem um valor de 01 a 07 quanto à importância de cada ítem, sendo calculada a média aritmética e o desviopadrão. A análise de estatística inferencial foi realizada através da análise de variância. Os resultados da análise descritiva e os testes de estatística inferencial evidenciaram que a equipe de enfermagem identificou, como importantes para a causalidade da UP, fatores de risco referentes tanto ao paciente quanto à estrutura institucional e ao processo de cuidar. Segundo a percepção da equipe, o paciente com traumatismos da medula espinhal apresenta diminuição ou perda da mobilidade e sensibilidade, tornando-se dependente de outra pessoa para mobilizar-se. A estrutura institucional e o processo de cuidar não atendem às necessidades do paciente, assim, não se faz a rigorosa mudança de decúbito como deveria ser feita, os colchões utilizados não são adequados, levando o paciente a uma permanência prolongada no leito, em uma só posição, o que causa excesso de pressão nas proeminências ósseas. Após a alta, o problema persiste, pois, segundo a percepção dos participantes da pesquisa, a família não tem conhecimento a respeito da prevenção e não presta cuidados adequados em casa. Quanto às medidas preventivas, houve diferença entre a percepção dos auxiliares/técnicos e a dos enfermeiros; estes últimos consideraram todos os descritores do questionário importantes para a prevenção, enquanto os auxiliares/técnicos não consideraram. Houve uma discrepância no que diz respeito aos fatores de risco, que os auxiliares/técnicos consideraram importantes e o que apontaram como intervenções para prevenir o problema.

UNITERMOS: Úlcera de Decúbito. Traumatismos da Medula Espinhal. Cuidados de Enfermagem.

*Trabalho desenvolvido com apoio do CNPq- Projeto de Iniciação Científica - PIBIC - 1998 - 2000. 


\section{INTRODUÇÃO}

O traumatismo da medula espinhal (TME) é um grande problema de saúde no Brasil e no mundo, afetando, com maior freqüência, indivíduos adultos e do sexo masculino. Acidentes automobilísticos, quedas, mergulhos e a violência, com ferimentos por arma de fogo, são citados como as causas mais freqüentes ${ }^{(1)}$.

Dados do IBGE, referentes ao censo de $1991^{(2)}$, demonstram que existem, no Brasil, ao redor de 200.000 paraplégicos e 46.000 tetraplégicos. Embora a causa do problema não seja identificada pelo censo, grande parte deles provavelmente, foi vítima de lesão traumática medular.

Em decorrência da perda da mobilidade e da sensibilidade abaixo do nível medular acometido, o indivíduo poderá apresentar uma série de complicações dentre elas: as genitourinárias, as gastrintestinais, as cardiocirculatórias, as pulmonares e as úlceras de pressão ${ }^{(3)}$.

A úlcera de pressão, dependendo do nível de profundidade da lesão nos tecidos pode trazer sérias complicações como a osteomielite, septicemia e, mesmo, levar o paciente a óbito. Além das perdas financeiras ocasionadas ao paciente e familiares, o problema traz, também, transtornos psicológicos e impedem ou dificultam a participação do indivíduo em programas de reabilitação ${ }^{(1)}$. Assim, a prevenção e tratamento no estágio inicial devem ser a meta da assistência que vise à qualidade ${ }^{(4,5)}$.

A manutenção da integridade de pele e tecidos subjacentes tem sido tradicionalmente uma responsabilidade da equipe de enfermagem, embora outros profissionais da equipe de saúde devam estar envolvidos pela natureza multicausal do problema ${ }^{(6)}$. A presença das úlceras de pressão tem sido apresentada como um indicador da qualidade de assistência dos serviços de saúde e a maior parte delas pode ser prevenida com a adoção de medidas adequadas e educação para prevenção dirigida a profissionais, pacientes e familiares $^{(4,6,7)}$.

A etiologia da úlcera é multidimensional, e os fatores apresentados como mais importantes são a imobilidade e a diminuição da percepção sensorial, que levam ao excesso de pressão e isquemia. Os fatores externos, como a fricção, cisalhamento e umidade da pele, irão afetar tanto as condições de resistência dos tecidos ao excesso de pressão quanto os fatores relacionados às condições nutricionais do indivíduo ${ }^{(6)}$.
A atuação da equipe de saúde na prevenção do problema foi destacada nas diretrizes para a prática clínica do órgão do governo norte-americano, "Agency for Heath Care Policy and Research"(7). Tais diretrizes foram construídas por um painel de especialistas e têm sido utilizadas no mundo todo para nortear a assistência dentro do modelo da Medicina Baseada em Evidências. As recomendações são feitas enfocando e discriminando as ações necessárias para a prevenção da úlcera de pressão dentro de quatro aspectos básicos: avaliação do risco do paciente e dos fatores que o colocam em risco; cuidados com a pele e tratamento precoce da úlcera; redução da carga mecânica pelo reposicionamento e utilização de superfícies especiais de suporte, como almofadas e colchões; educação de pacientes, cuidadores e fornecedores de serviços.

A prevenção e tratamento da úlcera de pressão no lesado medular também foi o enfoque de diretrizes construídas por um consórcio americano, composto de membros de associações profissionais e acadêmicas, na área da saúde, e o apoio financeiro e administrativo da Associação de Veteranos com Paralisia ${ }^{(4)}$. Essas diretrizes trouxeram o enfoque para os fatores de risco, relacionados a questões psicossociais, como os comportamentos, condições econômicas, estilo de vida e adaptação psicológica de pacientes e familiares.

Rangel \& Caliri, 1997, ${ }^{(5)}$ identificaram que $22,5 \%$ dos 31 pacientes com TME, atendidos em um hospital universitário, durante um período de três anos, apresentaram UP entre o $7^{\circ}$ e $37^{\circ}$ dia da primeira hospitalização após o acidente e que não havia uma padronização de medidas de prevenção ou tratamento do problema pela equipe que prestou assistência.

Para que haja uma redução dos índices de úlcera de pressão é necessário que os fatores de risco dos pacientes sejam conhecidos de forma que medidas preventivas, relacionadas ao problema sejam implementadas na instituição.

Specht; Bergquist \& Frantz, 1995(8), destacam que, para o desenvolvimento de protocolo de cuidados para a padronização da assistência, além das bases científicas fornecidas pela pesquisa, é necessário que se identifiquem a prática e o conhecimento dos indivíduos envolvidos, motivos pelos quais nos propusemos a desenvolver esta pesquisa. 


\section{OBJETIVO}

O estudo teve como objetivo:identificar, segundo a opinião de enfermeiros, auxiliares e técnicos de enfermagem de uma instituição hospitalar, os fatores de risco ou as causas de UP em pacientes com TME, que são por eles atendidos e quais as medidas eles identificam como mais importantes para a prevenção do problema na instituição.

\section{MATERIAL E MÉTODOS}

O estudo foi desenvolvido através de um inquérito conduzido com membros da equipe de enfermagem de uma instituição hospitalar de ensino universitário do Interior Paulista, após a aprovação pela comissão de ética e com o consentimento informado dos participantes. A seleção dos participantes em potencial foi feita, considerando-se os locais do Hospital das Clínicas de Ribeirão Preto que prestam atendimento a pacientes com lesão medular, em unidades de internação ou ambulatório. Na Unidade de Emergência, consideramos a Clínica Médica e Cirúrgica, a CTI e a Unidade de Ortopedia. No HC-Campus, consideramos a Clínica Cirúrgica, as unidades de Neurologia, Ortopedia e Ambulatórios de Cirurgia e Ortopedia. Os enfermeiros, auxiliares e técnicos de enfermagem das citadas unidades, que estavam nas escalas de serviço e presentes nas unidades no período estabelecido para a coleta de dados, foram convidados a participar da pesquisa, preenchendo pessoalmente o instrumento de coleta de dados. A amostra foi de conveniência ou acidental. Todos os funcionários contatados aceitaram participar, no entanto, do total de 105 questionários distribuídos, 85 foram devolvidos $(81 \%)$.
O instrumento de coleta de dados foi composto de duas partes: a primeira constou de informações de dados demográficos dos participantes e a segunda de 35 frases com descritores dos fatores de risco e 18 frases com descritores de medidas preventivas, e ele foi construído a partir de dados da literatura ${ }^{(9)}$ e validado anteriormente. Foi solicitado aos participantes que atribuíssem um valor de 01 a 07 quanto à importância de cada um dos 35 itens como fator de risco ou causa de UP e quanto aos 18 itens como medidas preventivas importantes para serem utilizadas na instituição.

Para as variáveis demográficas foram obtidas as freqüências absolutas e a porcentagens. A análise estatística descritiva foi realizada, utilizando-se o programa EXCEL. Para cada um dos fatores de risco e medidas preventivas, foi calculada a média aritmética e o desvio-padrão de forma a identificar a ordenação de posições (ranking) dos fatores de risco, segundo a importância atribuída pelos, grupos de enfermeiros e auxiliares/técnicos. Foram considerados importantes, pela análise descritiva, os fatores nos quais a média dos escores atribuídos foi acima de 4.0 e sem importância aqueles com média menor de 4.0. Para comparar os valores das médias aritméticas dos 35 fatores de risco e verificar se havia diferenças estatisticamente significativas entre elas, foi feita análise de variância (one way ANOVA) tanto para os resultados relacionados ao grupo de auxiliares/técnicos de enfermagem como para os enfermeiros. O nível de significância utilizado foi $\square=0.05$.

\section{RESULTADOS}

Tabelas.

\begin{tabular}{|c|c|c|c|c|c|c|}
\hline \multirow{2}{*}{$\begin{array}{l}\text { Categoria } \\
\text { Profissionalocubacional }\end{array}$} & \multicolumn{2}{|c|}{$\begin{array}{l}\text { Awxiliarfécnico } \\
\text { de erfermagem }\end{array}$} & \multicolumn{2}{|c|}{ Enfermeiro } & \multicolumn{2}{|c|}{ Total } \\
\hline & $n$ & $\%$ & $n$ & $\%$ & $n$ & \% \\
\hline Masculino & 20 & 23,53 & 1 & 1,18 & 21 & 24,71 \\
\hline Feminino & 45 & 52,94 & 19 & 22,35 & 64 & 75,29 \\
\hline Total & 65 & 76,47 & 20 & 23,53 & 85 & 100 \\
\hline
\end{tabular}




\section{DISCUSSÃO}

Participaram da pesquisa 85 profissionais de saúde, dentre os quais, 21 eram do sexo masculino e 64 do feminino (Tabela I). Quanto à categoria profissional/ocupacional, o maior contingente $(76,47 \%)$ foi representado por auxiliares/técnicos de enfermagem. Os resultados confirmam dados de pesquisa nacional anterior sobre o perfil da equipe de enfermagem, realizada pelo Conselho Federal de Enfermagem quando a maior parte da equipe foi do sexo feminino e da classe atendente/auxiliar de enfermagem, hoje substituída pelos auxiliares e técnicos em decorrência da extinção da categoria atendente de enfermagem pela Lei $\mathrm{n}^{0}$ 7.498/86 que regulamenta o exercício profissional ${ }^{(10)}$. Em relação à idade dos sujeitos (Tabela II), a maior parte tinha entre 35 e 40 anos $(32,94 \%)$. Na categoria de auxiliares/técnicos, observamos a presença de indivíduos com mais de 45 anos (13\% do grupo) o que não ocorreu na categoria dos enfermeiros. A maior parte dos participantes da pesquisa atuava no HC-Campus (14 enfermeiros e 51 auxiliares/ técnicos). Na Unidade de Emergência, o maior número de participantes atuava na clínica médico-cirúrgica (50\%). No HC-Campus, 35\% atuavam na Clínica Cirúrgica e 34\% na Ortopedia.

Dentre os 35 fatores de risco ou causas para úlcera de pressão, apresentados no instrumento, não foram encontradas diferenças entre atribuição de importância para os grupos de auxiliares e técnicos e enfermeiros. Os testes de estatística inferencial, en- tretanto, identificaram grupos diferentes de causas segundo o valor de importância atribuído pelos membros da equipe.

Na Tabela III, apresentamos os fatores de risco para úlcera de pressão, ordenados em posições ou "ranking", segundo a atribuição de importância para os enfermeiros e auxiliares/técnicos. Para os enfermeiros, em relação aos fatores de risco, quatro grupos distintos foram encontrados (grupos A, B, C e D), o que significa que atribuíram diferentes importâncias aos fatores apresentados na literatura para a causalidade da úlcera na instituição ( $\square \square 0,03)$. Entretanto, dentre cada um dos grupos,não houve um fator que foi considerado mais importante que outro ( $\mathrm{p} \sqsubset 0,05)$.

Para os auxiliares/técnicos de enfermagem também foram encontrados quatro grupos distintos (A, B, $\mathrm{C}$ e D) em relação às médias obtidas para os fatores de risco $(\mathrm{p} \square 0,05)$ e dentro de cada grupo também não houve um fator mais importante que outro ( $\mathrm{p} \sqsubset 0,05)$.

Para os dois grupos (enfermeiros e auxiliares/ técnicos), os 11 fatores que receberam as maiores médias (grupo A) foram aqueles referentes à fisiopatologia da úlcera, associados à dependência do paciente para cuidados especiais no hospital e, posteriormente, no domícilio. Observamos que a equipe de enfermagem, ao identificar tais fatores como muito importantes, estão reconhecendo que, na instituiçao estudada, os fatores principais para a etiologia do problema nos pacientes com lesão medular são aqueles referentes ao seu quadro clínico, no entanto, reconhecem, também, a presença de fatores negativos ligados ao am-

\begin{tabular}{|c|c|c|c|c|c|c|}
\hline \multicolumn{7}{|c|}{$\begin{array}{l}\text { Tabela Il: Distribuição dos participantes em relação à categoria profissional/ocupacional e à faixa etári } \\
\text { - Ribeiraáo Preto, } 2001 \text {. }\end{array}$} \\
\hline \multirow{2}{*}{$\begin{array}{c}\text { Categoria } \\
\text { Profissionavocupacional } \\
\text { idade-anos }\end{array}$} & \multicolumn{2}{|c|}{ Auxiliar/Técnico de enfermagem } & \multicolumn{2}{|c|}{ Enfermeiro } & \multicolumn{2}{|c|}{ Tota! } \\
\hline & $n$ & $\%$ & $n$ & $\%$ & $n$ & $\%$ \\
\hline $20 \dashv 25$ & 7 & 8,24 & 1 & 1,18 & 8 & 9,42 \\
\hline $25 \dashv 30$ & 5 & 5,88 & 7 & 8,24 & 12 & 14,12 \\
\hline $30 \dashv 35$ & 8 & 9,41 & 5 & 5,88 & 13 & 15,29 \\
\hline $35 \dashv 40$ & 23 & 27,06 & 5 & 5,88 & 28 & 32,94 \\
\hline $40 \dashv 45$ & 11 & 12,94 & 2 & 2,35 & 13 & 15,29 \\
\hline $45 \dashv 50$ & 6 & 7,06 & - & - & 6 & 7,06 \\
\hline$=50$ & 5 & 5,88 & - & - & 5 & 5,88 \\
\hline TOTAL & 65 & 76,47 & 20 & 23,53 & 85 & 100,00 \\
\hline
\end{tabular}


biente ou à estrutura de trabalho, como a falta de rigorosa mudança de decúbito e uso de colchão não adequado. Os profissionais perceberam, também como importante para a ocorrência da úlcera, a falta de cuidados adequados em casa e a falta de conhecimento da família para os cuidados preventivos. Assim, aos fatores intrínsecos e extrínsecos, relativos ao problema do paciente com lesão medular, seja o paraplégico ou o tetraplégico, são acrescentados aqueles referentes à estrutura do serviço e ao processo de cuidar.

Os resultados indicam que as opiniões dos profissionais, geradas a partir de suas experiências na assistência a pacientes com lesão medular, são semelhantes às afirmações encontradas nas publicações nacionais $^{(1,3,7)}$ e internacionais ${ }^{(4,6,7,11 / 14)}$.

Para Yarkony, 1994(11), as pessoas com TME têm o risco bem conhecido para úlcera de pressão e ele é mais elevado no período imediatamente após a lesão, devido à imobilização prolongada em superfícies duras, e está inversamente relacionado aos valores da pressão arterial sistólica.

Maklebust \& Magnan 1994(12) , analisaram 2189 casos de pacientes hospitalizados em uma instituição americana de nível terciário e encontraram que 12,3\% tinham úlcera de pressão. Fatores de risco, como imobilidade, incontinência fecal, alteração nutricional e diminuição da percepção sensorial, tinham uma elevada associação com a presença da úlcera $(\mathrm{p}<0,002)$. Também estavam associados a presença de doença vascular periférica, diabetes mellitus ou lesão da medula espinhal.

Niazi et al., $1997^{(13)}$, destacaram que $85 \%$ das pessoas com TME apresentam úlceras de pressão em algum momento de suas vidas e que 5 a $91 \%$ dos lesados medulares apresentam recidivas. Enfatizaram a necessidade de reavaliação do tratamento oferecido a esses pacientes e das medidas preventivas, utilizadas.

Salzberg et al.,1998 ${ }^{(14)}$,desenvolveram uma pesquisa para identificar as variáveis associadas com úlcera de pressão em veteranos de guerra com lesão medular, de diferentes etiologias. Os autores identificaram que os principais fatores preditivos para úlcera de pressão foram: nível de atividade e mobilidade, lesão medular completa, presença de incontinência e disreflexia autonômica. Concluíram que as pessoas com risco mais elevado precisam ser avaliadas de forma mais freqüente e participar de programas de prevenção.

Os 14 fatores de risco que compuseram o grupo B (Tabela III), embora tenham obtido menores mé- dias, também foram considerados fatores muito importantes pelos participantes da pesquisa. Neste grupo, encontramos, principalmente, questões relacionadas ao inadequado cuidado com o lesado medular e questões referentes às condições da instituição para que o cuidado seja de qualidade.

No grupo C, foram incluídos os 8 descritores ou fatores de risco considerados de menor importância, que obtiveram média abaixo de 4,0, a maior parte deles referente aos aspectos de déficit de conhecimento do paciente, dos profissionais e ocupacionais, além da falta de orientação e acompanhamento médico. Tais fatores, entretanto, são reconhecidos pelas diretrizes internacionais para prevenção de úlcera de pressão como de suma importância para a mudança da situação das instituições e têm recebido destaque nos programas de melhoria dos indicadores de qualidade dos serviços de saúde ${ }^{(15)}$.

Observamos que alguns fatores de risco, como presença de espasmos e o paciente estar com anemia, não foram valorizados pelos participantes da pesquisa como importantes, embora sejam relatados como associados com o aumento e o número de úlcera de pressão ${ }^{(4)}$.

Paciente com depressão foi o fator de risco apontado como o de menor importância para os profissionais/ocupacionais (grupo D), no entanto, pesquisas recentes ${ }^{(16)}$ têm destacado elevada prevalência de depressão em pessoas com lesão medular, principalmente na fase aguda da hospitalização, o que causa interferência em suas atividades de autocuidado e nas relações interpessoais com cuidadores; assim, o fator precisa ser considerado pela equipe. Também Yarkony, $1994^{(11)}$ destacou que a adaptação psicossocial afeta a capacidade de a pessoa seguir as recomendações feitas quanto aos cuidados com a pele. Relatou que as pessoas deprimidas e aquelas que têm a tendência para auto-negligência podem desenvolver úlcera de pressão por não atenderem as suas necessidades de autocuidado. O autor afirmou ainda que o cuidado precário no domicílio, as recomendações inadequadas de cuidadores e os cuidados inadequados, em hospitais gerais, por pessoas não familiarizadas com os princípios de cuidados com a pele para as pessoas com paralisia, podem contribuir para o desenvolvimento da úlcera.

Para Olshanky, 1994(17) a incidência da úlcera de pressão nos hospitais é diretamente proporcional ao nível de conhecimento e compromisso dos cuidadores e administradores com a prevenção, já que os 
Tabela III - Ordenação de posições (ranking) dos fatores de risco para úlcera de pressão em pacientes com
TME, segundo a atribuiçáo de importância para os enfermeiros e auxiliares técnicos de enfermagem. GRUPO A: maiores médias obtidas.

\begin{tabular}{|c|c|c|c|c|}
\hline \multirow{2}{*}{ Fatores de risco para o desewoivimento de úicera de pressäo } & Ranking & Grupo & Ranking & Grupo \\
\hline & Enfermeiro & \multicolumn{3}{|c|}{ Auxtécnico } \\
\hline Permanência prolongada no leito, em uma só posiçẫo & $1^{\circ}$ & A & $1^{\circ}$ & A \\
\hline Falta de mudança riqorosa de decúbito & $2^{\circ}$ & A & $3^{\circ}$ & A \\
\hline Longa permanência em superfícies duras & $3^{\circ}$ & A & $2^{\circ}$ & A \\
\hline Excesso de pressẫo nas proeminências ósseas & $4^{\circ}$ & A & $4^{\circ}$ & A \\
\hline Dependência de outra pessoa para mobilizaçẫo & $5^{\circ}$ & A & $5^{\circ}$ & A \\
\hline Falta de cuidados adequados em casa & $6^{\circ}$ & A & $6^{\circ}$ & A \\
\hline $\begin{array}{l}\text { Diminuiçãojperda da sensibilidade ou capacidade de sentir dor ou } \\
\text { desconforto. }\end{array}$ & $7^{\circ}$ & A & $8^{\circ}$ & A \\
\hline Falta de conhecimento da família para cuidados preventivos & $8^{\circ}$ & A & $7^{\circ}$ & A \\
\hline Perda ou diminuição da mobilidade & $9^{\circ}$ & A & $10^{\circ}$ & A \\
\hline Irritacẫo da pele por incontinência urinária ou fecal & $10^{\circ}$ & A & $11^{\circ}$ & A \\
\hline Uso do colchẫo nẫo adequado & $11^{\circ}$ & A & $9^{\circ}$ & A \\
\hline Hospitalização prolonqada com cuidado inadequado & $12^{\circ}$ & 日 & $14^{\circ}$ & $\mathrm{B}$ \\
\hline Friccẫo ou atrito do corpo com superfícies ásperas & $13^{\circ}$ & $\mathrm{B}$ & $12^{\circ}$ & $\mathrm{B}$ \\
\hline Falta de funcionários na unidade & $14^{\circ}$ & $\mathrm{B}$ & $13^{\circ}$ & $\mathrm{B}$ \\
\hline Falta de um proqrama de prevençẫo para o paciente e família & $15^{\circ}$ & $\mathrm{B}$ & $20^{\circ}$ & $\mathrm{B}$ \\
\hline Paciente vem de casa com escaralúlcera de pressẫo & $16^{\circ}$ & 日 & $16^{\circ}$ & 日 \\
\hline Paciente vem de outro hospital com escaraiúlcera de pressão & $17^{\circ}$ & $\mathrm{B}$ & $15^{\circ}$ & $\mathrm{B}$ \\
\hline $\begin{array}{l}\text { Falta de detec ção precoce de sinais indicativos de escaralúlcera de } \\
\text { pressẫo. }\end{array}$ & $18^{\circ}$ & $\mathrm{B}$ & $18^{\circ}$ & $\mathrm{B}$ \\
\hline Falta de fisioterapia & $19^{\circ}$ & 日 & $19^{\circ}$ & 日 \\
\hline $\begin{array}{l}\text { Movimentação do paciente de forma incorreta (puxar, arrastar ou } \\
\text { empurrar o paciente). }\end{array}$ & $20^{\circ}$ & $\mathrm{B}$ & $17^{\circ}$ & $\mathrm{B}$ \\
\hline Falta de cuidados e inspeçẫo da pele com freqüência & $21^{\circ}$ & $\mathrm{B}$ & $22^{\circ}$ & $\mathrm{B}$ \\
\hline Estado nutricional insatisfatório & $22^{\circ}$ & 日 & $21^{\circ}$ & $\mathrm{B}$ \\
\hline Assistência de enfermagem nẫo adequada & $23^{\circ}$ & $\mathrm{B}$ & $24^{\circ}$ & $\mathrm{B}$ \\
\hline Falta de alimentaçẫo adequada & $24^{\circ}$ & 日 & $23^{\circ}$ & 日 \\
\hline Falta de planejamento de cuidados preventivos durante a internacẫo & $25^{\circ}$ & 日 & $25^{\circ}$ & 日 \\
\hline Falta de conhecimento do paciente sobre a prevencẫo & $26^{\circ}$ & $\mathrm{C}$ & $26^{\circ}$ & $\mathrm{C}$ \\
\hline $\begin{array}{l}\text { Falta de avaliação do estado qeral de saúde do paciente pela } \\
\text { enfermagem. }\end{array}$ & $27^{\circ}$ & $\mathrm{C}$ & $27^{\circ}$ & $\mathrm{C}$ \\
\hline Falta de conhecimento da enfermagem & $28^{\circ}$ & $\mathrm{C}$ & $28^{\circ}$ & $\mathrm{C}$ \\
\hline $\begin{array}{l}\text { Pouco conhecimento dos profissionais sobre escaralúlcera de } \\
\text { pressấo e medidas preventivas. }\end{array}$ & $29^{\circ}$ & $\mathrm{C}$ & $29^{\circ}$ & $\mathrm{C}$ \\
\hline Falta de preparo para a alta hospitalar & $30^{\circ}$ & $\mathrm{C}$ & $32^{\circ}$ & $\mathrm{C}$ \\
\hline Falta de orientaçẫolacompanhamento médico & $31^{\circ}$ & $\mathrm{C}$ & $30^{\circ}$ & $\mathrm{C}$ \\
\hline Presenca de espasmos ou "repuxos" involuntários do corpo & $32^{\circ}$ & $\mathrm{C}$ & $31^{\circ}$ & $\mathrm{C}$ \\
\hline Paciente com anemia & $33^{\circ}$ & $\mathrm{C}$ & $33^{\circ}$ & $\mathrm{C}$ \\
\hline A escaral úlcera de pressẫo é inevitável nestes pacientes & $34^{\circ}$ & $\mathrm{D}$ & $34^{\circ}$ & $\mathrm{C}$ \\
\hline Paciente com depressẫo & $35^{\circ}$ & $\mathrm{D}$ & $35^{\circ}$ & $\mathrm{D}$ \\
\hline
\end{tabular}


pacientes em risco estão, na maior parte dos casos, em situação passiva, dependente do cuidado. Afirma que, nas instituições onde o compromisso é maior, a incidência da úlcera é menor e que a maior parte das úlceras ocorrem não por o paciente ter um risco elevado, mas, porque o cuidado não foi adequado, seja por falta de conhecimento, recursos humanos ou materiais. Cita estudos que evidenciaram que programas educacionais e fornecimento de condições adequadas de trabalho para os profissionais permitiram a diminuição das úlceras.

A importância do enfoque educacional nas intervenções direcionadas para pacientes e cuidadores /profissionais e familiares para a prevenção da úlcera de pressão, em pacientes em risco, foi ressaltada por Bergstrom et al., $1992^{(7)}$ e também recomendada para serem incorporadas na assistência a pacientes com lesão medular pelo "Consortium for Spinal Cord Medicine"(4). As afirmações são originadas de painéis de consenso entre especialistas para uma prática baseada em evidência, visando melhorar a qualidade da assistência prestada a pessoas com TME.

A avaliação da opinião dos participantes da pesquisa quanto as medidas necessárias para a prevenção do problema na instituição em que trabalham (Tabela IV) evidenciou que para os enfermeiros, todas as medidas apresentadas são de igual importância e todas receberam médias elevadas (Grupo A). Entretanto, para os auxiliares/técnicos de enfermagem, dois grupos distintos (A e B) foram encontrados $(p<0,05)$.

Dentre as medidas vistas como de maior importância para todos os participantes, encontramos a

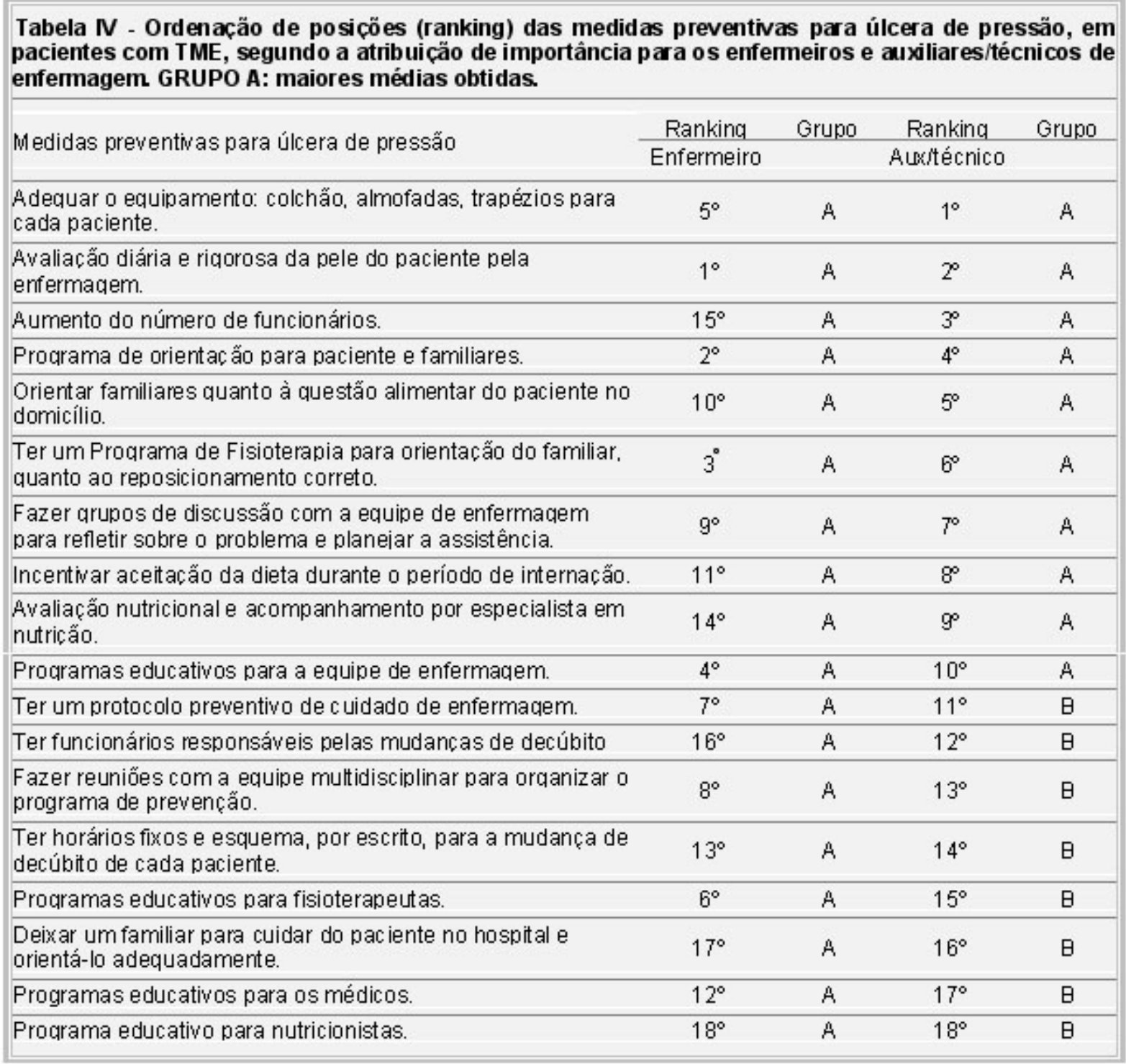


avaliação diária e rigorosa da pele do paciente pela equipe de enfermagem, o que confirma que os participantes identificam o lesado medular como sendo de risco elevado para apresentar a úlcera e valorizam a necessidade da avaliação para detectar a presença do problema, um aspecto enfatizado, na literatura, como prioritário na assistência preventiva e curativa e que deve ser realizado na admissão e de forma sistemática durante a internação ${ }^{(4,6)}$. Tal avaliação, entretanto, é de pouca valia se não for documentada no prontuário do paciente e se não gerar intervenções da equipe ao detectar o problema. A falta de documentação nos prontuários impede que estudos retrospectivos sejam realizados, além disso, pode sugerir que os profissionais estão sendo negligentes na assistência.

Dentre as medidas valorizadas como importantes pelos auxiliares/técnicos, encontramos aspectos referentes à estrutura institucional, como a adequação de equipamentos, aumento do número de funcionários e programas de orientação para pacientes e familiares com inclusão de outros membros da equipe de saúde como fisioterapeutas e nutricionistas, além da formação de grupos de discussão e programas educativos para a equipe de enfermagem. Entretanto, medidas, como programas educativos para nutricionistas/médicos e fiseoterapeutas assim como reuniões com a equipe multidisciplinar, para organizar o programa de prevenção não tiveram a mesma importância. mentos.

Os resultados levam-nos a alguns questiona-

Os auxiliares/técnicos acreditam que necessitam de maior conhecimento para a prevenção da úlcera de pressão? A menor valoração de programas de prevenção para outros membros da equipe multidisciplinar significa que, para eles, tais profissionais já têm formação necessária ou não devem ser responsáveis ou participar de atividades que visem à prevenção da úlcera?

As menores médias atribuídas às medidas, como ter um protocolo preventivo de cuidado de enfermagem, ter funcionários responsáveis pelas mudanças de decúbito e ter horários fixos e esquema por escrito para mudança de decúbito, teriam algum significado especial para os ocupacionais, como, por exemplo, o maior controle de suas atividades?

Os participantes do grupo deram uma menor atribuição de importância à estratégia de deixar um familiar no hospital e orientá-lo para o cuidado no domicílio, no entanto, esta é uma medida enfatizada nas diretrizes para a prevenção da úlcera como requisito básico para possibilitar o cuidado adequado à longo $\operatorname{prazo}^{(4,7)}$.

Quando comparamos os resultados referentes à valoração dos fatores de risco (Tabela III) e as medidas preventivas (Tabela IV), identificados como mais importantes pelos auxiliares/técnicos, observamos que há uma discrepância nas indicações o que pode significar um déficit de conhecimento dos mesmos sobre as intervenções adequadas para previnir a úlcera de pressão.

\section{CONSIDERAÇÕES FINAIS}

Através dos resultados obtidos, concluímos que, em relação à valoração da importância dos fatores de risco para UP, nos pacientes com TME, não houve diferenças significativas entre as opiniões dos auxiliares/técnicos e dos enfermeiros.Para eles, as úlceras de pressão no lesado medular ocorrem não somente em conseqüência dos fatores intrínsecos ao paciente e às conseqüências do trauma medular, mas, também, em decorrência da estrutura da instituição e do trabalho e do processo de cuidar. Verifica-se, assim, que, para prevenir o problema, torna-se necessário utilizar abordagens que lidem com todo o sistema, integrando o paciente e o ambiente institucional adequado. Porém, em relação as medidas preventivas, houve diferenças entre as categorias de profissionais. Enquanto os enfermeiros consideraram todas as medidas citadas como importantes, para os auxiliares/técnicos há diferenças na atribuição de importância às medidas preventivas. Houve uma discordância entre os fatores de risco considerados como mais importantes pelos auxiliares/técnicos de enfermagem e as medidas de intervenção que eles mais valorizaram como importantes para a prevenção do problema. Os achados indicam caminhos para novas pesquisas e apontam para a necessidade de intervenções educativas para todos os membros da equipe de enfermagem, para fornecer mais conhecimentos a respeito da prevenção do problema.

Acreditamos que a assistência ao lesado medular deva ter, como um dos objetivos a manutenção da integridade da pele e favorecer a cicatrização da úlcera presente e que a assistência deva ter uma base teórica consistente com as evidências científicas ou alicerçada no consenso de especialistas. Acreditamos que o uso apropriado das intervenções selecionadas trará um menor custo emocional e financeiro para o paciente, para a família e para o Sistema de Saúde. 
NOGUEIRA PC; CALIRI MHL \& SANTOS CB. Risk factors and prevention of pressure ulcer in spine cord injury patients. experience of members of nursing team at FMRP - USP Hospital Medicina, Ribeirão Preto, 35: 14-23, jan/march. 2002.

ABSTRACT: Pressure ulcers are frequent in spinal cord patients and can bring complication as osteomyelitis, sepses, amputation and even death. It interferes on quality of life of patients and families and brings difficulties for rehabilitation access. Being that, prevention or treatment in their initial stages needs to be the goals of a quality care. This study had as objectives to identify the causes of pressure ulcer in spinal cord injury patients and to describe prevention measures considered important as seem by professional nurses, nurses technicians and nurses auxiliaries, members of nursing team on an university hospital in Brazil. Through a survey, data was collected using an validated instrument containing 35 phrases descriptors of risk factors and 18 descriptors of preventive interventions. Each of 85 participants gave a value from 1 to 7 to the importance of the item and mean, standard deviation analysis of variance was calculated. The results of descriptive analysis and inferential statistics showed that the nursing team identified as important factors related to patient, to institutional structure and process of care. By their perception patients with spinal cord injury present immobility and impaired sensory perception, being dependent for mobilization. The process and structure of care does not respond to this need so the turning of patient is not rigorously done and bed mattress are not adequate what causes an excess of pressure on bone prominence. After discharge, the problem continues as families do not have enough knowledge and do not care adequately. Related to preventive measures there were differences between professional nurses and nurses technicians/auxiliaries. The former considered every measure as important but nurses technicians and auxiliaries did not and discrepancies were found between what they see as causes for pressure ulcer and what they consider important as preventive measures to minimize the problem.

UNITERMS: Decubitus Ulcer. Spinal Cord Injuries. Nursing Care.

\section{REFERÊNCIAS BIBLIOGRÁFICAS}

1 - FARO ACM. Do diagnóstico à conduta de enfermagem: a trajetória do cuidar na reabilitação do lesado medular. Tese de Doutorado, Escola de Enfermagem da USP, São Paulo p.1208,1995

2 - INSTITUTO BRASILEIRO DE GEOGRAFIAE ESTATÍSTICA. Censo demográfico 1991 http://www.sidra.ibge.gov.br

3 - SOUZA MITP. Úlcera de decúbito: história natural e informações de paraplégicos. Dissertação de Mestrado, Escola de Enfermagem de Ribeirão Preto da USP, Ribeirão Preto, p.1-102, 1988.

4 - CONSORTIUM FOR SPINAL CORD MEDICINE Pressure ulcer prevention and treatment following Spinal Cord Injury. A clinical practice guideline for health care professionals. Paralyzed Veterans of America, 2000.: http://www.pva.org.

5 - RANGEL EM \& CALIRI MHL. Prevalência de úlcera de pressão e medidas usadas para prevenção e tratamento em pacientes com lesão traumática de medula espinhal atendidos no HCFMRP- USP entre 1993 e 1995 - Relatório científico apresentado a FAPESP- Projeto de Iniciação Científica, Ribeirão Preto, dezembro, 1997, 36 p.

6 - MAKLEBUST J \& SIEGGREEN M. Pressure ulcer- Guidelines for prevention and nursing management- $2^{\text {nd }}$ ed, Spring House, Pennsylvania, 1996.
7 - BERGSTROM N.; ALLMAN RM; CARLSON CE; EAGLESTEIN W; FRANTZ RA; GARBER SL; GOSNELL D; JACKSON BS; KEMP MG; KROUSKOP TA; MARVEL EM; RODCHEAVER GT \& XAKELLIS GC. Pressure ulcers in adults: Prediction and Prevention. Clinical Practice Guideline. Quick Reference Guide for Clinicians. $n^{\circ} 3$ Rockville, MD: US Department of Health and Human Services, Public Health Service, Agency for Health Care Policy and Research. Publication $\mathrm{n}^{\circ}$ 92-0050. May, 1992.

8 - SPECHT JP; BERQUISTS \& FRANTZ RA. Adoption of a research based practice for treatment of pressure ulcers. Nurs Clin North Am 30: 553-563, 1995.

9 - NOGUEIRA PC \& CALIRI MHL. Fatores de risco para úlcera de pressão em pacientes com trauma medular. Análise da literatura científica nacional. $6^{\circ}$ Simpósio de Iniciação Científica da USP, Ribeirão Preto, novembro de 1998. v.1 p.97.

10 - BRASIL Lei n 7498 de 25 de junho de 1986. Dispõe sobre a regulamentação do exercício de enfermagem. Diário Oficial da União, 26 de junho de 1986, seção I, p. 9273 - 5, Brasília, DF, 1983.

11 - YARKONY GM. Pressure ulcers: a review. Arch Phys Med Rehabil 75:908-915, 1994.

12 - MAKLEBUST J \& MAGNAN M. Risk factors associated with having a pressure ulcer: a secondary data analysis. Adv Wound Care 7:25-42,1994. 
13 - NIAZI ZBM; SALZBERG CA; BYRNE DW \& VIEHBECK M. Recurrence of initial pressure ulcer in persons with spinal cord injuries. Adv Wound Care 10:38-42,1997.

14 -SALZBERG CA; BYRNE DW; CAYTEN CG; KABIR R; NIEWERBURGH P \& VIEHBECK M. Predicting and preventing pressure ulcers in adults with paralysis. Adv Wound Care 11:237-246,1998.

15 -FERNANDES LM. Úlcera de pressão em pacientes críticos hospitalizados. Uma revisão integrativa da literatura. Dissertação de Mestrado, Escola de Enfermagem de Ribeirão Preto da USP, Ribeirão Preto, p.1-168, 2000.
16 -KENNEDY P \& ROGERS BA. Anxiety and depression after spinal cord injury: a longitudinal analysis. Arch Phys Med Rehabil 81: 932-937, 2000.

17 -OLSHANKY K. Essay on knowledge, caring and psychological factors in prevention and treatment of pressure ulcer. Adv Wound Care 7: 64-68, 1994.

Recebido para publicação em 04/05/2001

Aprovado para publicação em 08/11/2001 